\title{
The NICA/MPD Project at JINR (Dubna)
}

\author{
Viacheslav Toneev * \\ JINR, Dubna \\ E-mail: toneev@theor.jinr.ru
}

\section{for the NICA/MPD working group}

A new project of Nuclotron-based Ion Collider fAcility (NICA) and Multi-Purpose Detector (MPD) is proposed at JINR (Dubna). Status of this NICA/MPD project is outlined. Main system parameters and some construction details are given.

Critical Point and Onset of Deconfinement - 4th International Workshop

July 9 - 13, 2007

Darmstadt, Germany

${ }^{*}$ Speaker. 


\section{Introduction}

The Joint Institute for Nuclear Research (JINR) in Dubna is an international research organization established in accordance with the intergovernmental agreement of 11 countries in 1956. At the present time, eighteen countries are the JINR Member States and five more countries have the associated member status. The JINR basic facility for high energy physics research is represented by the $6 \mathrm{AGeV}$ Nuclotron which has replaced the old weak-focusing $10 \mathrm{GeV}$ proton accelerator Synchrophasatron. The first relativistic nuclear beams with the energy of $4.2 \mathrm{AGeV}$ were obtained at the Synchrophasotron in 1971. Since that time the study of relativistic nuclear physics problems has been one of the main directions of the JINR research program.

The Nuclotron, $6 \mathrm{AGeV}$ synchrotron, based on unique fast-cycling supper-ferric magnets was designed and constructed at JINR (1987-1992) and commissioned in March 1993. The annual running time of 2000 hours was provided during the last years. Ion beams up to iron ions and polarized deuterons have been accelerated and extracted from the accelerator. The view of the Nuclotron ring in the tunnel is shown in Fig1.

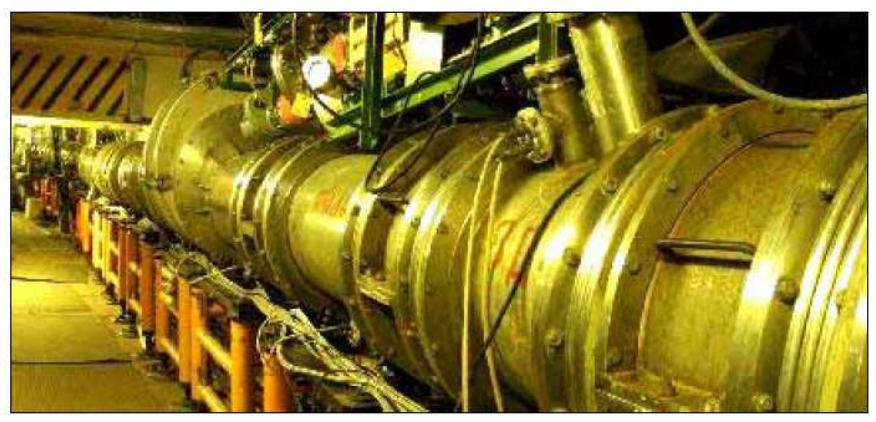

Figure 1: JINR Nuclotron

The new flagship of the Joint Institute for Nuclear Research is the NICA/MPD project to start experimental study of hot and dense strongly interacting matter at the new JINR facility [1] in the coming years. It may be reached by 1) Development of the existing Nuclotron accelerator facility as a basis for generation of intense beams over atomic mass range from protons to uranium and light polarized ions; 2) Design and construction of heavy ion collider (NICA) with maximum collision energy of $\sqrt{s}=9 \mathrm{AGeV}$ and average luminosity $10^{27} \mathrm{~cm}^{-2} \mathrm{~s}^{-1}$, and 3) Design and construction of a multi-purpose particle detector (MPD) at intersecting rings. Realization of the project will lead to unique conditions for the world community research activity. The NICA energy region is of great interest because the highest nuclear (baryonic) density under laboratory conditions can be reached there. Generation of intense polarized light nuclear beams aimed at investigation of polarization phenomena at the Nuclotron is foreseen. Future JINR facility was discussed at the Round Table meeting "Searching for the mixed phase of strongly interacting matter at the JINR Nuclotron" [2]. A conceptional project "Design and construction of the Nuclotron-based Ion Collider fAcilitiy (NICA and Multi-Purpose Detector (MPD) was first presented and discussed along with external experts at the Round Table II in October 2006 [3].

The program of the Nuclotron upgrade in the NICA context is in progress. The ions up to $\mathrm{Au}$ have been obtained at test bench based on the unique technology of highly charged state ion 
sources. Modernization of the Nuclotron is one of the key points in the NICA realization. We are planning the completion of the first stage work by fall of 2009 .

\section{NICA/MPD goals and physics problems}

Such an investigation is relevant for understanding the evolution of the Early Universe after Big Bang, formation of neutron stars, and physics of heavy ion collisions. The new JINR facility will make it possible to study in-medium properties of hadrons and nuclear matter of the equation of state, including a search for possible signatures of deconfinement and/or chiral symmetry restoration phase transitions, and QCD critical endpoint in the region of $\sqrt{\mathbf{s}_{\mathrm{NN}}}=3-\mathbf{9 ~ G e V}$ by means of careful scanning in beam energy and centrality of excitation functions. As is seen from Fig.2, the chosen energy interval indeed covers the most attractive domain of the phase diagram.

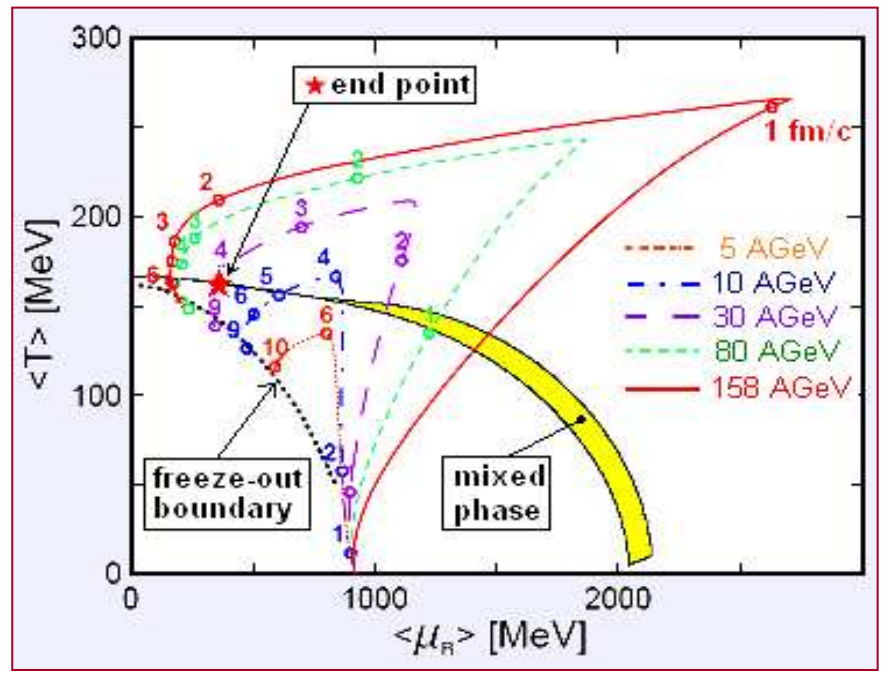

Figure 2: Dynamical trajectories for central $(b=2 \mathrm{fm}) \mathrm{Au}+\mathrm{Au}$ collisions in the $T-\mu_{B}$ plane for various bombarding energies calculated within the relativistic 3-fluid hydrodynamics with hadronic EoS [4]. Numbers near the trajectories are the evolution time moments. Phase boundary is estimated in a two-phase bag model taking into account the conservation of baryon and strangeness charges [5]. The critical end point calculated in the lattice QCD [6] is marked by the star.

The first stage measurements include:

- Multiplicity and global characteristics of identified hadrons, including multi-strange particles;

- Fluctuations in multiplicity and transverse momenta;

- Directed and elliptic flows for various hadrons;

- HBT and particle correlations.

Electromagnetic probes (photons and leptons) are supposed to be added at the second stage of the project. The beam energy of the NICA is very much lower than the energy region of the RHIC and 


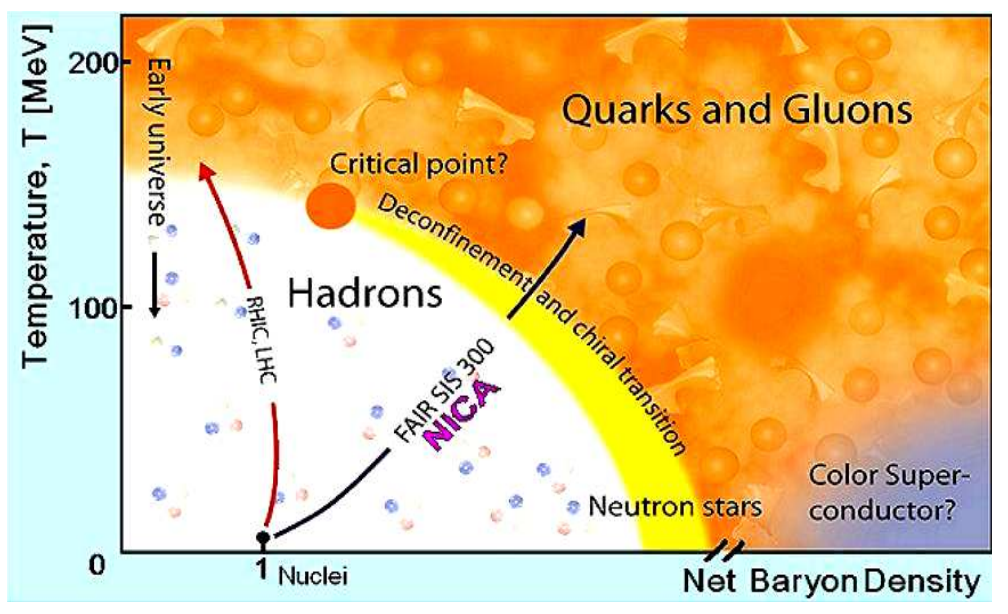

Figure 3: Phase diagram (Artist's view).

LHC but it sits right on the top of the region where the baryon density at the freeze-out is expected to be the highest. In this energy range the system occupies a maximal space-time volume in the mixed quark-hadron phase (the phase of coexistence of hadron and quark-gluon matter similar to the water-vapor coexistence phase). The net baryon density at LHC energies is predicted to be lower. The energy region of NICA as well as of FAIR will allow analyzing the highest baryonic density under laboratory conditions (see the diagram in Fig.3). These conditions are expected to be also at the FAIR facility in Darmstadt in 2015 when the synchrotron SIS300 will come into operation.

\section{NICA general layout}

Construction of the new facility is based on the existing buildings and infrastructure of the Synchrophasotron/Nuclotron of the JINR Veksler-Baldin Laboratory of High Energies. The accelerator chain includes (see Fig.4): Heavy-ion source - Linac - Booster ring - Nuclotron - Superconduct-

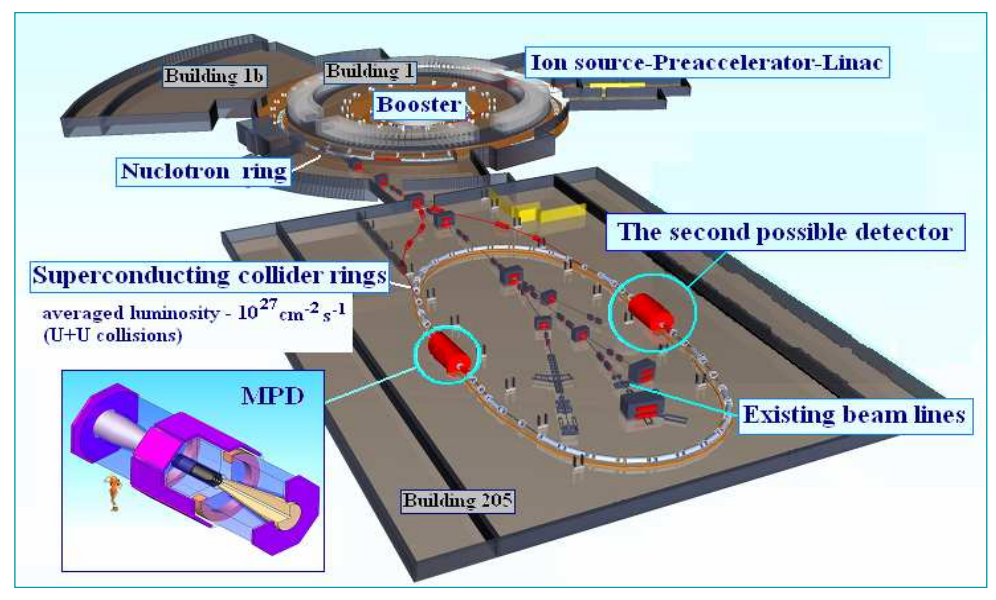

Figure 4: General layout of the NICA/MPD 


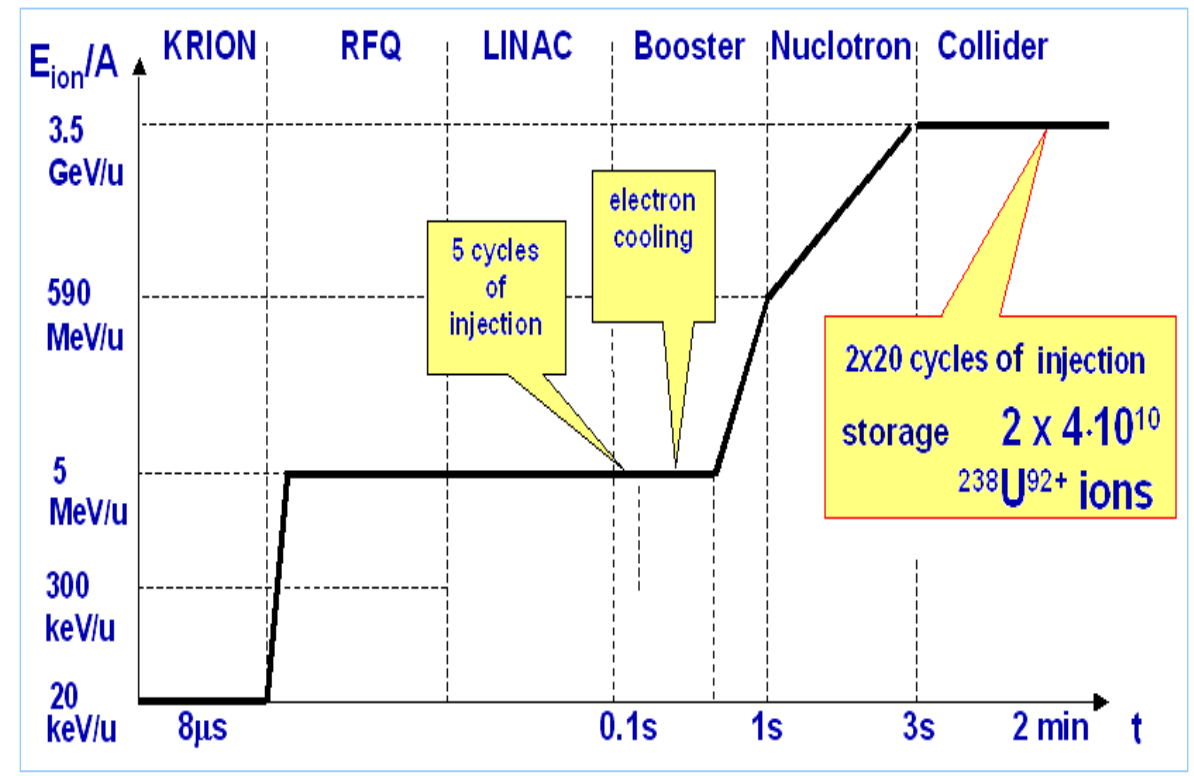

Figure 5: The NICA time scheme

ing collider rings. The peak design kinetic energy of ${ }^{238} U$ ions in the collider is $3.5 \mathrm{AGeV}$. Beam cooling and bunching systems are foreseen. The collider magnetic system is fitted to the existing buildings. The project design presumes the use of some fixed target experiments. A polarized deuteron beam from the Nuclotron will be available too. The collision mode is under discussion. Several schemes of the NICA complex have been considered since 2006. This one under careful analysis at the present time is presented below.

\begin{tabular}{|l|r|}
\hline Ring circumference, $m$ & 251.2 \\
Ion kinetic energy, $E[\mathrm{GeV} / u], \mathrm{min} / \mathrm{max}$ & $1 / 3.5$ \\
Particle number per bunch, $N_{\text {ion } / \text { bunch }}$ & $2.0 \cdot 10^{9}$ \\
Bunch number, $n_{\text {bunch }}$ & 20 \\
Horizontal emittance, $\varepsilon[\pi \mathrm{mm} \mathrm{mrad}]$ & 0.7 \\
Momentum spread, $\Delta p / p$ & 0.001 \\
IBS life time. $[\mathrm{sec}]$ & $\geq 100$ \\
Beta function at interaction points, $\beta^{*}[\mathrm{~m}]$ & 0.5 \\
$\mathrm{RF}$ voltage, $U_{R F}[\mathrm{kV}]$ & 200 \\
Laslett tune shift, $\Delta Q$ & 0.0044 \\
Beam-beam parameter & 0.009 \\
Luminosity, $L\left[\mathrm{~cm}^{-2} \mathrm{~s}^{-1}\right]$ peak/average & $2 /(1-1.5) \cdot 10^{27}$ \\
\hline
\end{tabular}

Table 1: The main NICA parameters

Operation scenario is illustrated by the diagram in Fig.5. During the first $8 \mu \mathrm{sec}$ the KRION source produces the ion beam with energy $20 \mathrm{KeV} / u$ which is quickly accelerated by RF Quadruples till $5 \mathrm{MeV} / u$ and then after LINAC is injected into Booster. Here the beam is cooled down electronically and is accelerated again reaching the energy of $590 \mathrm{MeV} / u$ by the time of $1 \mathrm{sec}$ com- 


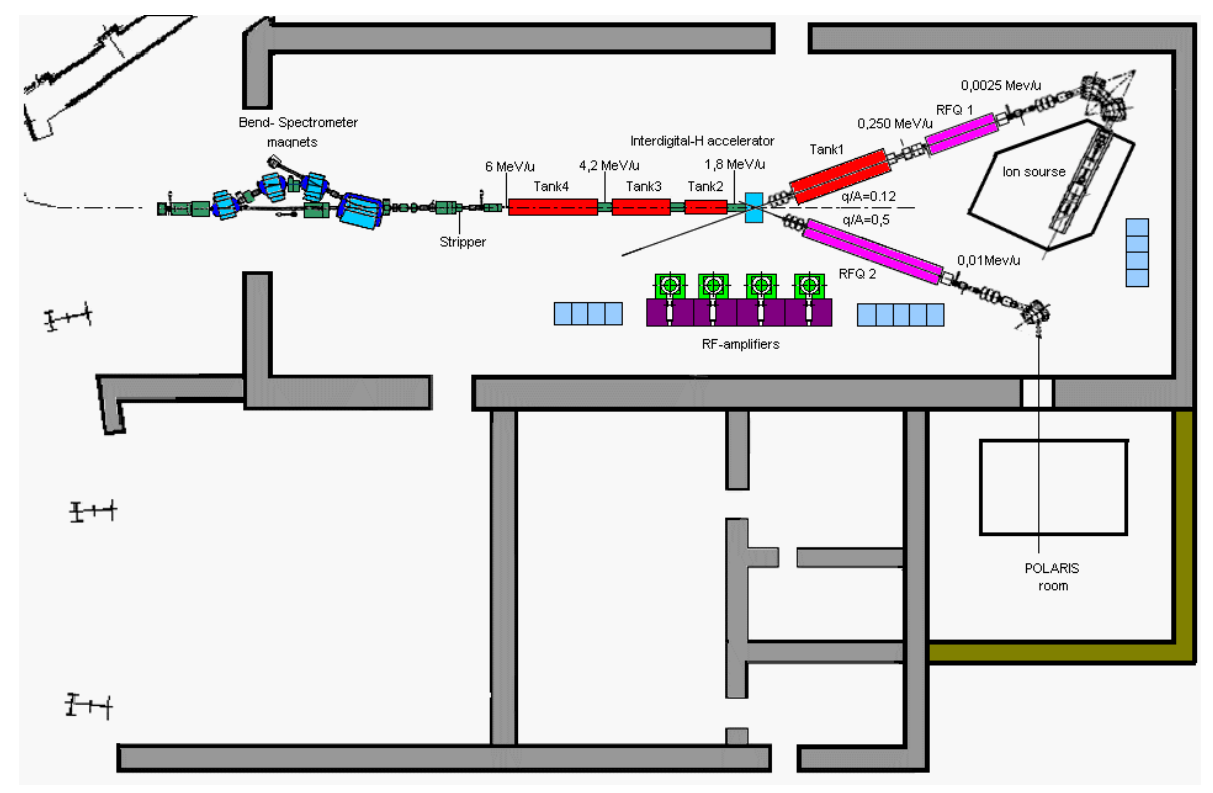

Figure 6: Injector scheme

pleting the pre-injection stage. Now the beam may be accelerated in the Nuclotron to the maximal project energy $3.5 \mathrm{GeV} / \mathrm{u}$ and 20 cycles storage in every Collider Ring for about $200 \mathrm{sec}$ to be compatible with the Intra Beam Scattering (IBS) lifetime. These $2 \times 20$ cycles of injection result in $2 \times 4 \cdot 10^{10}$ ions. The expected parameters of the collider are presented in Table 1 . The specified average luminosity of $1 \cdot 10^{27} \mathrm{~cm}^{-2} \mathrm{~s}^{-1}$ can be reached at the chosen parameters.

Let us consider the constituent elements of the NICA system in more detail. The whole injection system is shown schematically in Fig.6. Its heart element, the KRION ion source, was created at JINR [7] and, as is seen from Table 2, has a very significant advantage in the main parameter, as compared to the ECR source: A number of ions per unit time is higher by factor 5 in the KRION source. Note that for the KRION all numbers in this table are measured experimentally.

\begin{tabular}{|l|c|c|}
\hline Ion source & $\mathrm{KRION}, A u^{30+}$ & $\mathrm{ECR}, \mathrm{Pb}^{27+}$ \\
\hline Peak ion current $[\mathrm{mA}]$ & 1.2 & 0.2 \\
Pulse duration $[\mu \mathrm{sec}]$ & 8 & 200 \\
Ions per pulse & $2 \cdot 10^{9}$ & $1 \cdot 10^{10}$ \\
Ions per $\mu \mathrm{sec}$ & $2.5 \cdot 10^{8}$ & $5 \cdot 10^{7}$ \\
Norm. rms emittance & $0.15-0.3$ & $0.15-0.3$ \\
Repetition rate & 60 & 30 \\
\hline
\end{tabular}

Table 2: Comparison of ion source parameters

The Injector consists of Linac structures, RFQ and RF generator (see Fig.6) and, in addition, Diagnostic, Control and Water cooling systems which will be manufactured at IHEP (Serpukhov) and assembled at JINR. A similar system has been constructed by IHEP for CERN (see Fig.7). The cost of the whole equipment is $10 \$ M$.

We consider the possibility to use a space inside the Synchrophasotron magnet yoke window 


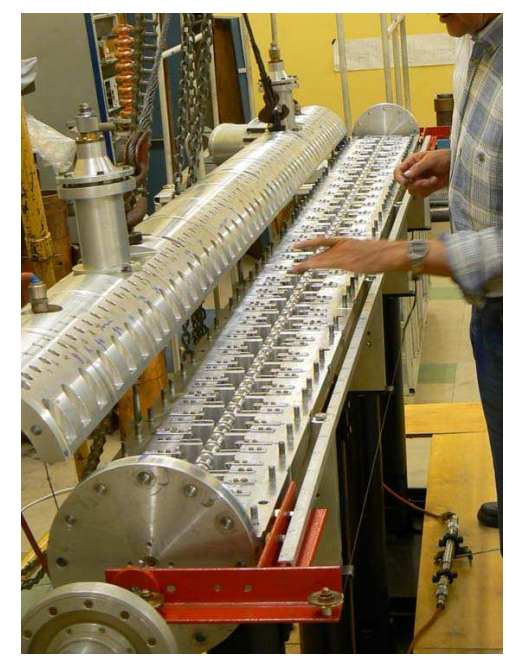

Figure 7: Injector element at IHEP

for installation of the booster synchrotron, as is shown in Fig.8. The "warm" Booster will have 70 dipole magnets of $1.37 \mathrm{~T}$ max magnet field. A cost estimate of the booster is about $8 \$ M$. It will be manufactured by the Central Machinery Workshop of JINR.
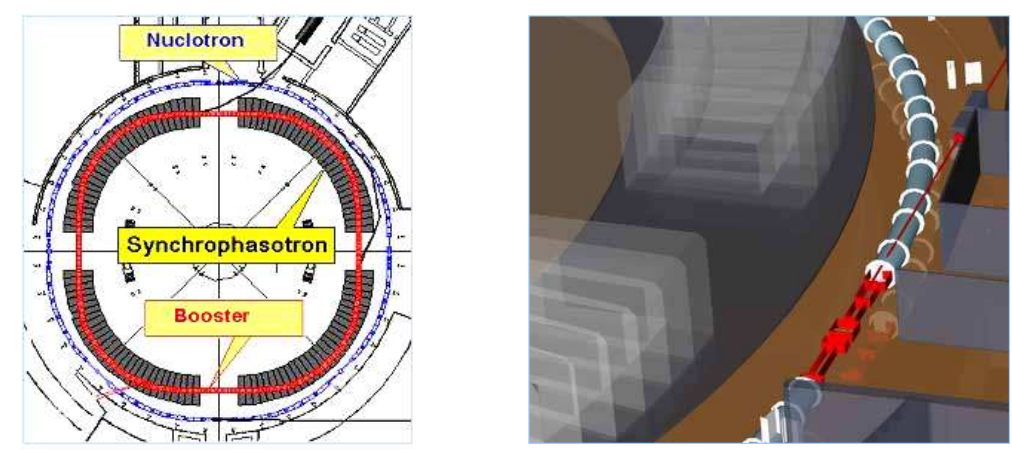

Figure 8: Schematic (left) and pictorial (right) view of the booster.

The booster will fill two Collider Rings of about $250 \mathrm{~m}$ circumference. These rings have two intersecting points where detectors will be installed.

Note that the project has essential cost saving factors: no new buildings, no additional power lines, and no extra heat, water, cooling power are needed.

So, a NICA cost estimate (in $\$ M$ ): KRION+HV "platform" - 0.25, Injector (IHEP design) 10, Booster -8 , Collider Rings $-2 \times 10$, i.e. totally $\sim 40 \$ M$.

\section{MPD for mixed-phase experiments}

The experimental set-up of proposed MPD has to detect the high multiplicity events and perform particle identification. The MPD scheme presented in Fig.9 indicates general elements of a typical collider detector which is at the initial stage of conceptional design. The tracking system, both the inner detector based on silicon strips (Silicon Vertex Tracker) and the Tracker provide the 
reconstruction of primary and secondary vertices and precise measurement of particle momenta. The Tracker includes - Central Tracker Detector (CTD) and Forward Tracking Systems (FTS). Two alternative possible versions are considered as the CTD: the TPC and Straw Tracker (ST). All tracking detectors are installed in the magnetic field of $\sim 0.5 T$ which is parallel to a beam direction. For the particle identification Time of Flight (TOF) System based on the RPC is proposed. This system allows pion, kaon and proton identification in the momentum region $0.2-2 \mathrm{GeV} / \mathrm{c}$. The TPC option of the tracker could provide in addition particle identification by measuring its ionization energy loss $(d E / d x)$. For electron-positron and gamma detection an Electromagnetic Calorimeter (ECal) is considered for the central region of two Forward ECal's for high pseudo-rapidity. Two options for these calorimeters are under consideration: Crystal or Sandwich of plastic and lead multi-layers. Two near the beam sets of scintillator counters are used as start devices for TOF measurements and on-line vertex positioning. The two Zero Degree Calorimeters (ZDC) provide the energy measurement of spectators and determination of "centrality" in the ion-ion collision.

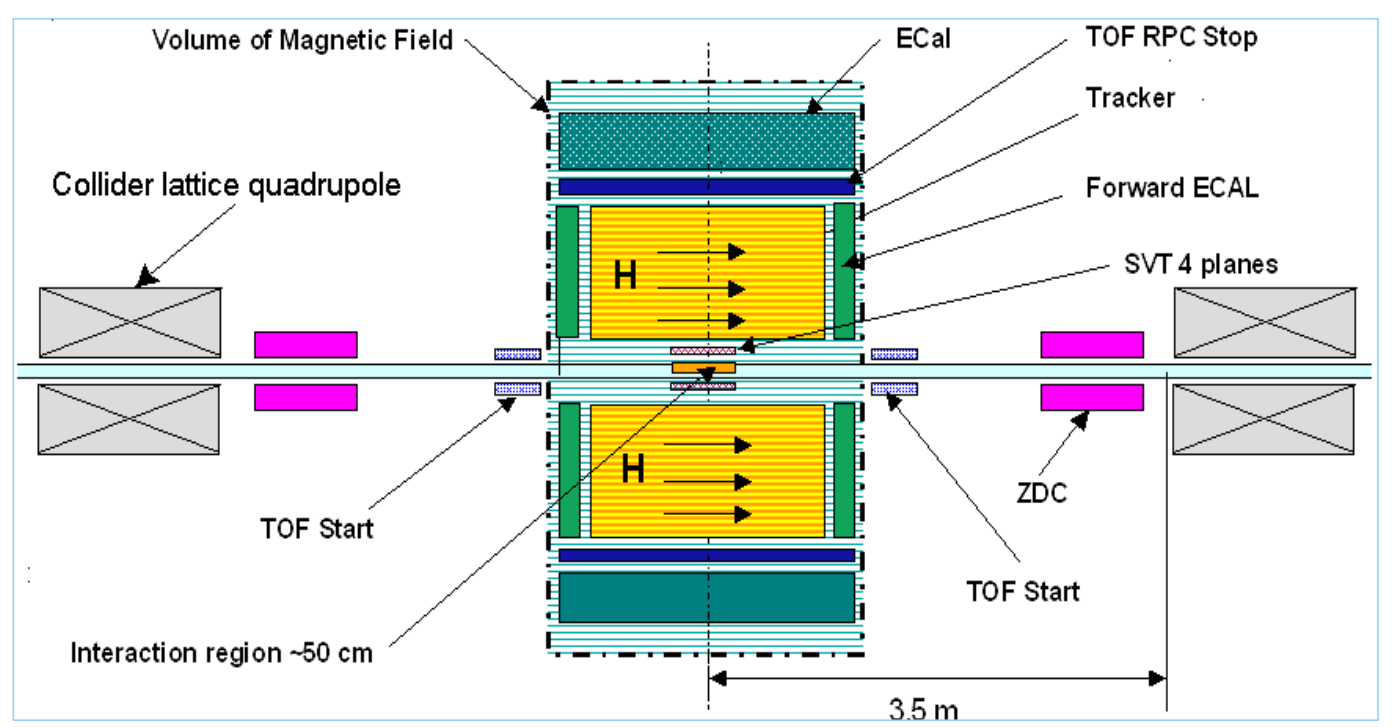

Figure 9: General scheme of the MPD.

General requirements to the detecting MPD system are the following: $|y|<2$ acceptance and $2 \pi$ continuous azimuthal coverage; high tracking efficiency; adequate track length for tracking, momentum measurement and particle identification; two track resolution providing a momentum difference resolution of a few $\mathrm{MeV} / \mathrm{c}$ for HBT correlation studies; the fraction of registered vertex pions $>70 \%$.

Some basic parameters of the setup:

- Interaction rate of $U+U$ events at luminosity of $10^{27} \mathrm{~cm}^{-2} \mathrm{~s}^{-1}$ is $10 \mathrm{kHz}$;

- Interaction rate of central events is $500 s^{-1}$ (It is assumed that DAC system is capable of reading-out and storing these data);

- The accuracy of vertex positioning by means of the silicon vertex detector is better than 0.2 $m m$; 

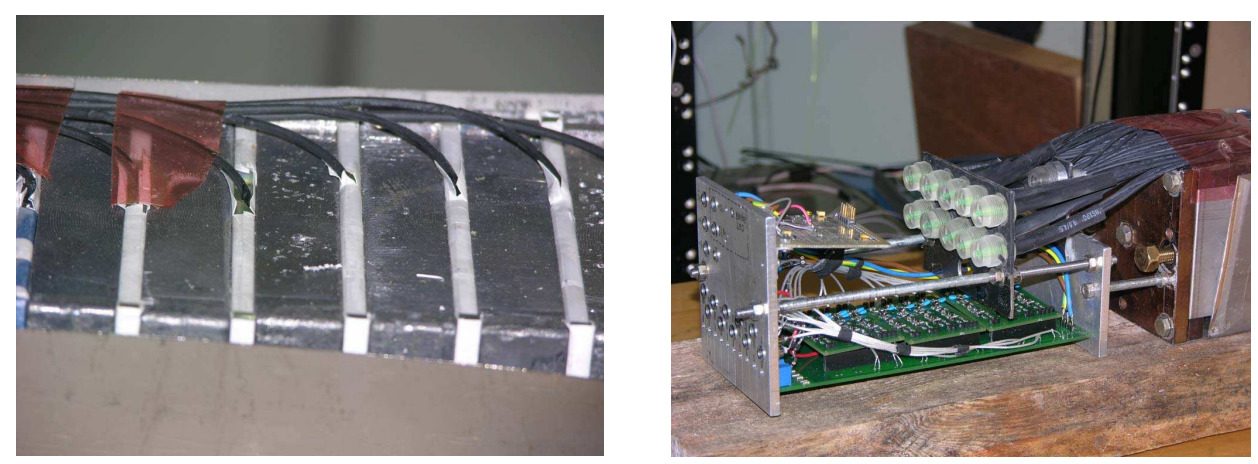

Figure 10: Elements of the ZDC at INR (Troitsk)

- The TPC produces 30 hits on a track and provides momentum measurement accuracy of $\sim 1 \%$ in the range of $p=0.2-2 \mathrm{GeV} / c$;

- The RPC time of flight system has the time resolution of $100 \mathrm{ps}$ and provides $\pi-K$ separation with probability $5 \%$ for $p<2 \mathrm{GeV} / \mathrm{c}$. At low momenta $p<0.7 \mathrm{GeV} / \mathrm{c}$ all particle identification is achieved by ionization measurement in TPC.

To select events, according to the centrality of a nucleus-nucleus collision, Zero Degree Calorimeters will be constructed. They will be made by INR (Troitsk) similarly to that manufactured for FAIR (see Fig.10).

A crude estimate of the MPD cost (without Zero Degree Calorimeter one) is about $25 \$ M$. In particular, it includes the cost (in $\$ M$ ) of Silicon vertex detector - 4.8, Time projection chamber 5, TOF system - 4, and EM calorimeter borrel - 3.5.

\section{Summary and outlook}

The proposed stages of the NICA/MPD project realization are the following:

- Stage 1: years 2007-2009

- Development of the Nuclotron facility

- Preparation of the Technical Design Report

- Start of prototyping of MPD and NICA elements

- Stage 2: years 2008-2011

- Design and construction of the NICA and MPD

- Stage 3: years 2011-2012

- Assembling

- Stage 4: year 2013

- Commissioning 
The total cost of the NICA/MPD project is about $70 \$ M$.

Finally, the new facility at JINR in Dubna, the NICA/MPD, will make it possible to study very important unsolved problems of strongly interacting matter. The design and organization work has been started. The Project Coordination Committee is established. A special scientific department for preparation of the project is formed. The first task of the NICA/MPD, the Conceptional Design Report, is planned to be completed by this fall. We suppose a wide-world cooperation with many Laboratories at both the R\&D and construction stages of work.

\section{Acknowledgments}

This work was supported in part by RFBR Grants N 05-02-17695 and 06-02-0400 as well as by the special program of the Ministry of Education and Science of the Russian Federation (grant RNP.2.1.1.5409).

\section{References}

[1] A.N. Sissakian, A.S. Sorin and V.D. Toneev, QCD Matter: A search for a mixed quark-hadron phase, nucl-th/0608032.

[2] Round Table Discussion I, Searching for the mixed phase of strongly interacting matter at the JINR Nuclotron, http://theor.jinr.ru/meetings/2005/roundtable/.

[3] Round Table Discussion II, Searching for the mixed phase of strongly interacting matter at the JINR Nuclotron: Nuclotron facility development, http://theor.jinr.ru/meetings/2006/roundtable/.

[4] Y.B. Ivanov, V.N. Russkikh and V.D. Toneev, Relativistic heavy-ion collisions within 3-fluid hydrodynamics: Hadronic scenario, Phys. Rev. C 73 (2006) 044904 [nucl-th/ 0503088 ].

[5] A.S. Khvorostukhin, V.V. Skokov, V.D. Toneev and K. Redlich, Lattice QCD constraints on the nuclear equation of state, Eur. Phys. J. C 48 (2006) 531 [nucl-th/ 0605069 ].

[6] Z. Fodor and S.D. Katz, Lattice determination of the critical point of QCD at finite T and $\mu, J H E P$ 203 (2002) 14 [hep-lat / 0106002 ]; Critical point of QCD at finite $T$ and $\mu$, lattice results for physical quark masses, JHEP 404 (2004) 50 [hep-lat / 0402006 ].

[7] E.D. Donets, 25 years with EBIS, Phys. Scripta T71 (1997) 5; M. Kleinod, R. Becker, H. Bongers, M. Weidenmuller, B. Zipfel and E.D. Donets, A generation of unconventional electron beam ion sources, Rev. Sci. Instrum. 67 (1996) 986. 